\title{
Genetic Basis for Clinical Response to CTLA-4 Blockade
}

TO THE EDITOR: Snyder et al. (Dec. 4 issue) ${ }^{1}$ state that in patients with melanoma who clinically benefit from blockade of cytotoxic T-lymphocyte antigen 4 (CTLA-4), the tetrapeptide sequences in tumor neoantigens were identical to those in known antigenic peptides in pathogens. The authors probably correctly interpret that this coincidence reflects cross-reactivity between cancer neoepitopes and relevant epitopes from the microbial counterparts. However, another explanation is possible. It could be that there was a positive selection in the thymus for rearranged T-cell receptors (TCRs) that preferentially recognize pathogen-encoded and major histocompatibility complex (MHC)-presented antigenic epitopes. Such a feature could have been sculpted by evolution in TCR variable genes and HLA genes.

More information about these interpretations could be gained by knowing the serologic status of the patients with respect to the putatively cross-reacting microbes. For instance, it would be very interesting to ascertain whether the serum samples from patient CR9306 were positive for anti-hepatitis D virus antibodies. Negative serologic status does not completely exclude previous subclinical contact with the infectious agent but would certainly argue against crossreactivity as an explanation for these observations.

\section{Ignacio Melero, M.D., Ph.D.}

Juan J. Lasarte, Ph.D.

\author{
Centro de Investigación Médica Aplicada \\ Pamplona, Spain \\ imelero@unav.es
}

Dr. Melero reports receiving consulting fees from BristolMyers Squibb, AstraZeneca, and Roche and research grants from Pfizer and Bristol-Myers Squibb. No other potential conflict of interest relevant to this letter was reported.

1. Snyder A, Makarov V, Merghoub T, et al. Genetic basis for clinical response to CTLA-4 blockade in melanoma. N Engl J Med 2014;371:2189-99.

DOI: $10.1056 /$ NEJMc1415938

THE AUTHORS REPLY: The similarities between pathogen antigenic peptides and peptide se- quences in tumor neoantigens in patients with melanoma who have a response to anti-CTLA-4 therapy may indeed arise from several factors. TCR cross-reactivity is one possibility, as described by Birnbaum et al. ${ }^{1}$ We agree that another possibility is that positive selection in the thymus and sculpting by evolution in TCR and HLA genes may have helped to mold the motifs. Indeed, Su et al. noted that pathogen-specific $\mathrm{T}$ cells may be abundant even in unexposed adults. ${ }^{2}$ Thus, in our patients, one can envision several possibilities. First, there may be common T-cell-recognition motifs present in T cells that underlie immunotherapy response regardless of exposure. Second, the patients who have a response to anti-CTLA-4 therapy may have been previously exposed to organisms with antigens homologous to tumor neoantigens and may have undergone priming. Or third, both of these hypotheses may be true. To answer these important questions, it will be necessary to characterize the serologic status of our patients for potential cross-reactivity with microbial antigens.

Important issues need to be addressed moving forward. At this juncture, the field of cancer immunogenomics and the use of high-level genetic analysis to decipher immune-related phenotypes are accelerating rapidly. Application of such efforts may lead to valuable insights in both immunology and cancer therapy.

\section{Alexandra Snyder, M.D. \\ Jedd D. Wolchok, M.D., Ph.D. \\ Timothy A. Chan, M.D., Ph.D.}

Memorial Sloan Kettering Cancer Center

New York, NY

chant@mskcc.org

Since publication of their article, the authors report no further potential conflict of interest.

1. Birnbaum ME, Mendoza JL, Sethi DK, et al. Deconstructing the peptide-MHC specificity of T cell recognition. Cell 2014;157: 1073-87.

2. Su LF, Kidd BA, Han A, Kotzin JJ, Davis MM. Virus-specific CD4(+) memory-phenotype $\mathrm{T}$ cells are abundant in unexposed adults. Immunity 2013;38:373-83.

DOI: 10.1056/NEJMc1415938 